\title{
Video Article \\ Use of Magnetic Resonance Imaging and Biopsy Data to Guide Sampling Procedures for Prostate Cancer Biobanking
}

\author{
Susan Heavey ${ }^{1}$, Aiman Haider ${ }^{2}$, Ashwin Sridhar ${ }^{3}$, Hayley Pye ${ }^{1}$, Greg Shaw ${ }^{1}$, Alex Freeman ${ }^{2}$, Hayley Whitaker ${ }^{1}$ \\ ${ }^{1}$ Molecular Diagnostics and Therapeutics Group, University College London \\ ${ }^{2}$ Research Department of Pathology, University College London \\ ${ }^{3}$ Department of Uro-Oncology, UCLH NHS Foundation Trust
}

Correspondence to: Susan Heavey at s.heavey@ucl.ac.uk

URL: https://www.jove.com/video/60216

DOI: doi:10.3791/60216

Keywords: Medicine, Issue 152, prostate, MRI, PEOPLE, biopsy, biobank, sampling, tissue, cancer, prostatectomy, slicing, genomics, culture

Date Published: 10/10/2019

Citation: Heavey, S., Haider, A., Sridhar, A., Pye, H., Shaw, G., Freeman, A., Whitaker, H. Use of Magnetic Resonance Imaging and Biopsy Data to Guide Sampling Procedures for Prostate Cancer Biobanking. J. Vis. Exp. (152), e60216, doi:10.3791/60216 (2019).

\section{Abstract}

Previous methods for biobanking prostate tissue, following radical prostatectomy, generally involved random sampling. In order to increase efficiency, and enable a greater range of downstream applications, a more targeted method of sampling prostate tissue was developed. Here we use both magnetic resonance imaging (MRI) and biopsy data to target specific areas of the organ for sampling. The method involves use of a previously published prostate slicing device which removes a $5 \mathrm{~mm}$ transverse slice from a predetermined region of the prostate, followed by the removal of $6 \mathrm{~mm}$ punch biopsies from predetermined areas of this slice. These samples can be stored frozen or fixed for biobanking purposes, or used fresh immediately with $70 \%$ confidence of tumor content, as compared with $10 \%$ confidence from the random sampling approach. This enables the use of all standard downstream techniques such as genomics, proteomics or histological work, but also work that requires fresh tissue such as live tissue imaging or ex vivo culture.

\section{Video Link}

The video component of this article can be found at https://www.jove.com/video/60216/

\section{Introduction}

Access to high quality human prostate cancer tissue is a key requirement for driving effective research in the field. There are a number of existing methods to sample prostate tissue following radical prostatectomy for research. Typically these involve using punch biopsies to take random samples from a fresh, frozen or fixed slice of prostate tissue, and retrospectively confirming whether or not tumor is present in each sample by hematoxylin \& eosin (H\&E) as assessed by a uropathologist ${ }^{1,2,3,4}$. A recent review has compiled an overview of these existing methods ${ }^{6}$. These methods are useful for certain downstream applications, where tissue can be stored and assessed for tumor content at a later date, such as large scale genomic analyses like the International Cancer Genome Consortium (ICGC) and The Cancer Genome Atlas (TCGA), However, these methods could be improved upon in if we were to use magnetic resonance imaging (MRI) and/or biopsy data to target specific areas of the prostate for sampling. This would improve the methodology in two ways; firstly, by reducing the number of tissue samples collected, increasing efficiency and reducing pressure on pathology departments and cost of storage, and secondly, by allowing fresh tissue to be used immediately without the need for immediate confirmation of tumor content, for new state of the art downstream technologies such as live tissue imaging, organoid generation or ex vivo culture. This research need has led to the development of the PEOPLE (PatiEnt prOstate samPLes for rEsearch) method, and the results from the first 84 cases biobanked using PEOPLE were recently published ${ }^{8}$. A variation of this method has also been published with a three-dimensional (3D) printed slicing apparatus and patient-specific mold, in order to facilitate ex vivo MRI on pre- and post-fixation tissue ${ }^{9,10}$.

\section{Protocol}

The protocol adheres to local guidelines and is approved by the UCL/UCLC Biobank Research Ethics Committee (Reference 15/YH/0311).

NOTE: As this method involves the sampling of human tissue, all local procedures regarding ethics and consent must be observed in advance of beginning the protocol. Radical prostatectomy cases may be included if both MRI and biopsy data are available in advance of surgery, with tumor diameter $\geq 5 \mathrm{~mm}$. Cases should be excluded if the index lesion is not well defined, i.e., only diffuse changes are visible by MRI.

\section{Prostate Slicing Apparatus}

1. Purchase the prostate slicing apparatus (Table of Materials). Alternatively, print a blade handle using a 3D printer as previously published ${ }^{10}$. 
NOTE: The device and disposable blades used here were purchased under material transfer agreement from the Institute of Cancer Research, London, UK.

\section{Tumor Targeting}

1. Review clinical notes to identify the index lesion as indicated by diagnostic biopsy, e.g., left posterior.

2. Review MRI images to measure location of the above tumor.

1. Find the sequence where the tumor is most visible in the axial plane, e.g., T2-weighted.

2. Scroll through axial images to find the image where the tumor is largest and print image for reference.

3. In the corresponding coronal image, measure the distance from the base of the prostate to the selected axial position, and the full length of the prostate from apex to base $(\mathrm{mm})$, and print for reference.

\section{Collection of pProstate}

1. Check patient notes to ensure appropriate informed consent has been obtained for this procedure and any downstream research applications.

2. Following radical prostatectomy, collect the prostate in a dry pot. Ensure no formalin or other fixative has been added to the prostate.

3. Transfer to a suitable sterile location for sampling, e.g., a laminar flow hood in a pathology laboratory.

4. Proceed to sampling as soon as possible if fresh tissue is required. NOTE: For certain applications (e.g., assessment of DNA which should not degrade as quickly as RNA), it may be appropriate to refrigerate the specimen and take samples the next day.

\section{Specimen Preparation}

1. Prepare laminar flow hood and prostate slicing apparatus according to local decontamination procedures, using sterile technique. Here, spray $70 \%$ ethanol and wipe across all surfaces. Use sterile single-use needles and scalpels. Use slicer blades up to three times; wash after each use in hot soapy water, then spray and wipe with $70 \%$ ethanol.

2. Weigh the prostate $(\mathrm{g})$ using a standard scale.

3. Ink the prostate. Paint the left side with blue ink and right side with black ink. Cover the full capsule and seminal vesicles with ink to later denote the surgical margins.

NOTE: Inking procedures may vary locally and can be modified accordingly.

\section{Prostate Slicing}

1. Assemble the slicing apparatus by inserting the walls perpendicularly into the base of the stand (Figure 1A).

2. Place prostate so that the base and apex are facing opposite walls, with the posterior side down and anterior up. Place gold pins around prostate. Push prostate inwards slightly if necessary to get a snug fit, which will support the prostate during slicing.

3. Measure prostate length from base to apex, using a ruler, and compare with prostate length as measured by MRI. If the prostate has shrunk, apply an ad hoc correction to the anticipated distance from base to target transverse slice. For example, if the full length of the prostate in the MRI image is $50 \mathrm{~mm}$, but when measured with a ruler at this point it has shrunk to $45 \mathrm{~mm}$, reduce the anticipated slicing position by $10 \%$.

4. Measure from the base to the desired transverse slice. Choose the pin that sits closest to this measurement to slice around.

5. Wearing chainmail gloves to prevent injury, hold slicing device (Figure 1B), place blades either side of the identified pin and use the spacer to keep blades $5 \mathrm{~mm}$ apart. Take slice by slowly and firmly moving the blades downwards, forwards and backwards with long strokes (Figure 1C). Ensure a full slice has been separated before disassembling apparatus.

6. Remove walls and pins and carefully take the slice out onto a sterile sheet of cork board using gloves.

\section{Tissue Sampling}

1. Visually inspect the transverse slice and compare with the axial MRI image. In some cases, the tumor area may appear paler than surrounding tissue.

2. Palpate the transverse slice gently. In some cases, the tumor may feel firmer than the surrounding tissue.

3. Using the axial MRI image as a guide, select one or more areas for sampling.

4. Take biopsy punches of desired area of tissue.

1. Using a $6 \mathrm{~mm}$ punch, push down on the desired area of tissue.

2. Twist the tissue punch on the spot and down against the cork to ensure full separation and use a sharp scalpel to separate if necessary.

3. Remove the punch and place into tubes/molds as necessary by ejecting using the plunger.

4. Repeat for further tumor and benign samples as required, with separate sterile biopsy punches. Ink the holes where punches were taken in red.

5. Note the location of each punch along with the weight of the prostate and any observations on tissue color/firmness. 


\section{Submission of Prostate for Local Diagnostics}

1. Pin the prostate to cork with sterile single-use needles prior to fixation in order to prevent tissue shrinkage and warping, which could alter the appearance of the surgical margins.

2. Following pinning to cork, submit the prostate to the histopathology department for standard clinical diagnostics.

\section{Decontamination of Apparatus}

1. Discard all disposable equipment in biomedical waste streams and/or sharps containers as designated locally.

2. Decontaminate the laminar flow hood and prostate slicing apparatus in accordance with local risk assessments appropriate to human tissue (e.g., by spraying with $70 \% \mathrm{EtOH}$ and wiping).

\section{Representative Results}

Fresh prostate tissue sampled using the PEOPLE method can be used for a variety of downstream techniques, including genomic sequencing and ex vivo culture. The first 59 cases sampled using this method have been previously published in comparison with an earlier version of the


$1 \mathrm{~min}$, which was kept to a minimum to avoid degradation of RNA. Time from removal of the prostate to prostate slicing should also be kept to a minimum, though here this took approximately $20 \mathrm{~min}$ due to our theatre and pathology labs being in different locations.

Depending on the downstream application, typically at least two samples are taken: one from an area of anticipated tumor tissue and one from an area of anticipated benign tissue. The key measure of success for the sampling method itself is to assess the tumor content in a given sample.

For entry into the 100,000 genomes project, an H\&E stained tissue section must be assessed by a uropathologist, and the sample must contain at least $40 \%$ tumor cells. Samples that contain less than $40 \%$ tumor may still be included in the project if they are successfully macrodissected. Of the first 92 cases sampled in this manner, $64 \%$ contained at least $40 \%$ tumor and were submitted to the 100,000 Genomes Project without macrodissection. DNA was extracted and was of sufficient yield and quality in all cases (Table 1). An initial subset of 59 of these samples was previously published in comparison with an earlier method $^{8}$.

For ex vivo culture, matched tumor and benign tissue must be of sufficient quality to withstand $72 \mathrm{~h}$ culture without significant degradation. Multiple tissue samples from a total of three patients were cultured successfully ${ }^{8}$.
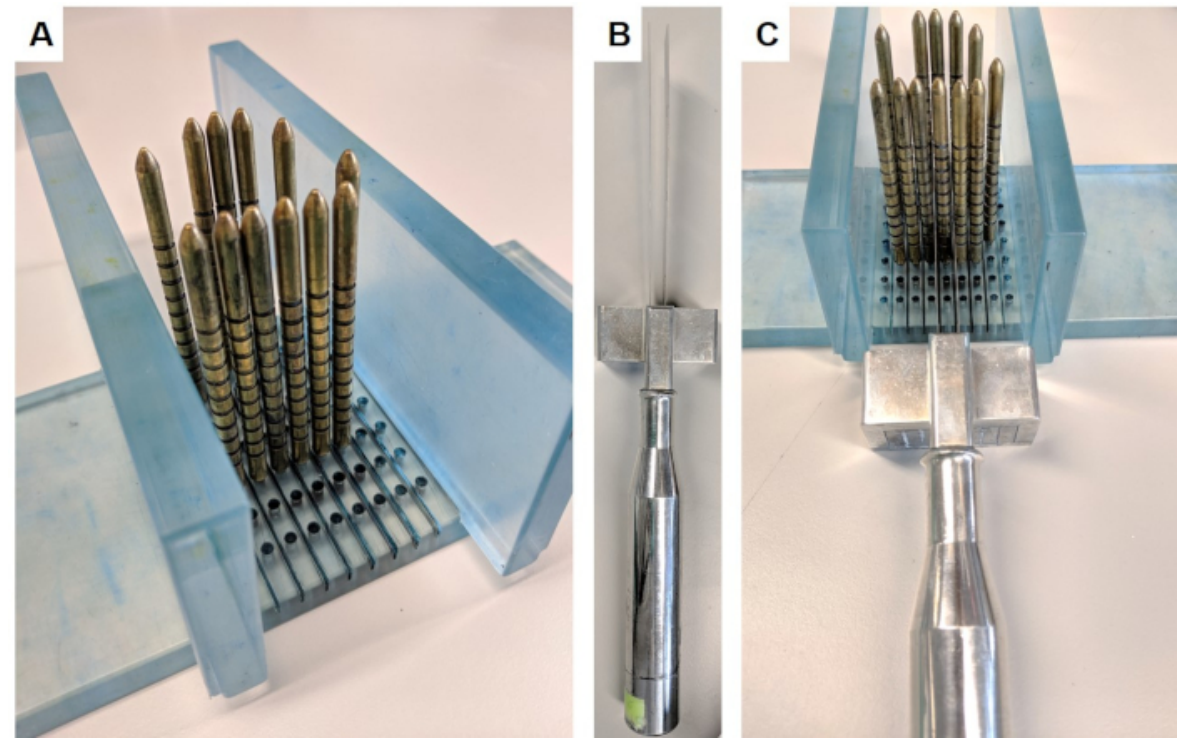

Figure 1: Prostate slicing apparatus. This apparatus was obtained under material transfer agreement from the Institute of Cancer Research. (A) The walls are inserted perpendicular to the base, and gold pins are inserted into the base surrounding the prostate (prostate not pictured). (B) The replaceable parallel blades are inserted into the blade handle. (C) The blades pass between the gold pins in order to slice a $5 \mathrm{~mm}$ section of the prostate. Please click here to view a larger version of this figure.

\begin{tabular}{|l|l|}
\hline & n (\%) \\
\hline Hit (>40\% tumor) & $59(64 \%)$ \\
\hline Partial hit (5-30\% tumor) & $6(7 \%)$ \\
\hline Miss (0\% tumor) & $27(29 \%)$ \\
\hline Total & $92(100 \%)$ \\
\hline
\end{tabular}


Table 1: Tumor hit rate. Tumor hit rate was determined by a consultant pathologist specializing in prostate cancer, following review of H\&E stained tissue. Tumor cell content of $>40 \%$ was determined to be suitable for inclusion in the 100,000 Genomes Project, as per Genomics England guidelines.

\section{Discussion}

Critical steps within this protocol include identification of tumor region for sampling, measurement of prostate, and tissue sampling. Firstly, measurement of the MRI to identify the correct area of the sampling is key. We demonstrate this method in the accompanying video; however, we also recommend confirming measurements with a radiologist in the first instance. Clear clinical notes which point the researcher towards the area of the MRI images that contains the index lesion are ideal. Secondly, measurement of the prostate should be carried out with care, ensuring that the ruler is held at an angle to measure the full length from base to apex, parallel to the anterior of the prostate. Thirdly, tumor areas should be confirmed prior to sampling by visually inspecting the tissue slice in relation to the original MRI image, palpating the tissue (in some cases the tumor area can feel more dense), and visually assessing the color of the tissue (in some cases the tumor will appear more pale than surrounding benign tissue).

This protocol has been carried out in full at UCL/UCLH by non-clinical postdoctoral researchers, a pathology fellow, pathology consultants, and research technicians. In our experience all steps of the protocol can be learned in under ten cases regardless of technical background. However, we recommend training from a radiologist regarding MRI measurement and training from a pathologist regarding slicing in the first instance. The protocol can be modified by using a 3D printed slicing handle, as previously published ${ }^{10}$.

Potential limitations of the technique include the risk of impeding on diagnosis. Slicing the prostate is a key step, which could impede on grading or positive margin rates if done incorrectly. There are two potential issues here. Firstly, if all of the index lesion is removed and used for fresh tissue experimentation immediately, routine clinical diagnostics will not be carried out for this lesion and the patient may be misdiagnosed as having a lower grade cancer. To avoid this, the researcher should discuss the sampling plan with the consultant pathologist who will routinely review the case, prior to sampling, and agree on the number, and location, of samples to be taken. Small tumors may be excluded locally for this reason. Secondly, if the prostatic capsule is not pinned down correctly to cork board prior to fixation, this could allow the inner tissue to bulge outwards during fixation, altering the surgical margins. This could lead to a false positive margin, where the remaining tumor appears to reside at the capsule purely due to tissue warping.

The significance of this technique with respect to existing methods lies mainly with tumor targeting. A range of methods for sampling radical prostatectomy specimens has been published to date; however, these all rely on a fully or partially random sampling approach ${ }^{1,2,3,4,5,6,7}$. The use of biopsy and in particular MRI data here has improved efficiency, allowing for reduced sampling with increased confidence of obtaining tumor tissue . $^{8}$.

Future applications of this method allow for the adoption of a wider range of downstream techniques than with previous sampling methods. For example, the availability of fresh tissue that has a high probability of being tumor means that more expensive and/or labor-intensive fresh tissue techniques can be utilized, as many samples are not required to ensure the presence of tumor. This can include and is not limited to, ex vivo culture, ex vivo MRI, advanced imaging and transcriptomics.

\section{Disclosures}

The authors have nothing to disclose.

\section{Acknowledgments}

The authors wish to acknowledge Prostate Cancer UK for funding SH under the Prostate Cancer UK Centre of Excellence and Travelling Prize Fellowship (TLD-PF16-004) and HP under INNOVATE (PG14-018-TR2). This work was supported by researchers at the National Institute for Health Research University College London Hospitals Biomedical Research Centre.

\section{References}

1. Sooriakumaran, P., Henderson, A., Denham, P., Langley, S.E. A novel method of obtaining prostate tissue for gene expression profiling. International Journal of Surgical Pathology. 17 (3), 238-243 (2009).

2. Jhavar, S.G. et al. Processing of radical prostatectomy specimens for correlation of data from histopathological, molecular biological, and radiological studies: a new whole organ technique. Journal of Clinical Pathology. 58 (5), 504-508 (2005).

3. Wheeler, T.M., Lebovitz, R.M. Fresh tissue harvest for research from prostatectomy specimens. The Prostate. 25 (5), $274-279$ (1994).

4. Esgueva, R. et al. Next-generation prostate cancer biobanking: toward a processing protocol amenable for the International Cancer Genome Consortium. Diagnostic Molecular Pathology. 21 (2), 61-68 (2012).

5. Dev, H. et al. Biobanking after robotic-assisted radical prostatectomy: a quality assessment of providing prostate tissue for RNA studies. Journal of Translational Medicine. 9, 121 (2011).

6. Tolkach, Y. et al. Blind Biobanking of the Prostatectomy Specimen: Critical Evaluation of the Existing Techniques and Development of the New 4-Level Tissue Extraction Model With High Sampling Efficacy. The Prostate. 77 (4), 396-405 (2017).

7. Warren, A.Y. et al. Method for sampling tissue for research which preserves pathological data in radical prostatectomy. The Prostate. 73 (2), 194-202 (2013).

8. Heavey, S. et al. PEOPLE: PatiEnt prOstate samPLes for rEsearch, a tissue collection pathway utilizing magnetic resonance imaging data to target tumor and benign tissue in fresh radical prostatectomy specimens. The Prostate. 79 (7), 768-777 (2019). 
9. Bailey, C. et al. VERDICT MRI validation in fresh and fixed prostate specimens using patient-specific moulds for histological and MR alignment. NMR in Biomedicine. 32 (5), e4073 (2019).

10. Bourne, R.M. et al. Apparatus for Histological Validation of In Vivo and Ex Vivo Magnetic Resonance Imaging of the Human Prostate. Frontiers in Oncology. 7, 47 (2017). 